\title{
Gender differences in time from arrival to stroke team activation in patients presenting with acute stroke symptoms
}

\author{
Kevin A Kotkowski ${ }^{1, \star,}, \odot$, Evangelia Murray ${ }^{1,2, \dagger, \oplus}$, Martin A Reznek ${ }^{1, \dagger}, \odot$, Sean S Michael ${ }^{1,2, \dagger, \oplus}$
}

${ }^{1}$ University of Massachusetts Medical School, Department of Emergency Medicine, 55 Lake Ave N, Worcester, MA 01655, USA

${ }^{2}$ University of Colorado School of Medicine, Department of Emergency Medicine, 12401 E 17th Ave \#B-215, Aurora, CO 80016, USA

\section{*Correspondence}

Kevin.kotkowski@umassmemorial.org (Kevin A Kotkowski)

${ }^{\dagger}$ These authors contributed equally.

\begin{abstract}
Background and Purpose: Women are more likely to experience delays in evaluation and treatment for acute stroke. As national guidelines increasingly emphasize the importance of rapid stroke intervention, it is important to further investigate this gender disparity. We sought to evaluate whether door-to-stroke activation time varied by patient gender among patients for whom stroke team activation occurred in the emergency department (ED).

Methods: This was a retrospective analysis of a prospectively collected registry of all patients for whom the stroke team was activated in the ED of an urban, academic, regional stroke center over 1 year. Our primary outcome was door to stroke activation time (DTA), with the primary predictor of interest being patient gender. We assessed for differences in DTA using multivariable Cox proportional hazards and logistic regression models.

Results: There were 211 patient encounters included in the study, 117 women and 94 males. Median DTA was 8 minutes longer for women, and women were less likely to have DTA $\leq 15$ minutes (odds ratio 0.26 [ $95 \%$ confidence interval 0.12 to 0.58 ]).

Conclusion: A gender disparity existed in door to activation time for women presenting with suspected acute stroke, even when controlling for a variety of factors. These results provide evidence that gender may impact initial management of stroke patients.
\end{abstract}

\section{Keywords}

Emergency medicine; Neurologic emergencies; Neurology; Gender disparities

\section{Introduction}

National guidelines emphasize rapid stroke identification and intervention in reducing stroke-related morbidity and mortality [1-3]. Women are known to experience delays in evaluation [4-9], receive fewer interventions such as thrombolytics [1013], and have more functional impairment after an acute stroke $[11,12,14,15]$, even when accounting for a number of confounding factors. Gender disparities in the ED components of acute stroke care appear to exist, but they are incompletely characterized to date.

Several studies have reported longer ED door-to-doctor or door-to-imaging (DIT) times for women [5, 8, 9, 15-21]. However, these studies vary in their methodology and particularly in their consideration of potential confounders. Notably, only one prior study incorporated ED triage when considering a potential relationship between gender and door-to-doctor and door-to-imaging time [22] but did not find gender to impact door-to-imaging time, which is in contrast to nearly all other prior reports $[5,8,9,15,18]$.

Potential gender disparities in acute stroke care may be amenable to intervention, and they warrant further investiga- tion, particularly in regard to contributing and confounding factors. Furthermore, prior studies have not considered doorto-stroke team activation time, which has been shown to significantly impact the likelihood of timely imaging and subsequent care $[6,22]$. Therefore, we sought to incorporate a broader list of known potential confounders to evaluate whether door-tostroke activation time, and secondarily, door-to-imaging time, varies by patient gender among patients without a pre-hospital stroke activation.

\section{Methods}

The data that support the findings of this study are available from the corresponding author upon reasonable request.

\subsection{Study design and setting}

This was an observational cross-sectional study in which we retrospectively queried the prospective stroke registry of our urban, regional referral stroke center hospital, which included all consecutive patients who presented to the adult ED between June 15, 2014 and June 14, 2015 for whom the stroke team 
was activated. To investigate gender differences in care, we employed a previously developed methodology to investigate a novel subset of the stroke registry [6]. During the study period, there were 67,795 total adult ED visits and approximately 27,000 adult inpatient admissions. The center is a primary teaching site for multiple residencies, including emergency medicine and neurology, and there is a stroke team available in-house 24 hours per day, 7 days a week. The stroke team may be activated prior to hospital arrival by emergency medical services (EMS) or at any time during triage or ED care by ED nurses or physicians. The ED is staffed by board-certified/board-eligible attending emergency physicians who supervise emergency medicine and off-service rotating residents. All American Heart Association/American Stroke Association Get With The Guidelines recommendations have been implemented [23, 24], and ED nursing and physician staff undergo periodic acute stroke continuing education. The study was approved by our Institutional Review Board.

\subsection{Selection of participants}

The institution maintains a prospective registry of all patients for whom the stroke team is activated, which includes patient demographics and time stamps for key care events including ED arrival time, stroke team activation time, imaging initiation and completion times, and time to thrombolytic administration. A stroke nurse coordinator maintains the registry and verifies its accuracy based upon established institutional guidelines. Automated and manual processes exist to ensure $100 \%$ registry capture of all patients for whom stroke resources are activated.

By protocol, the stroke team was activated when any patient presented to the ED with symptoms or findings consistent with an acute stroke within 12 hours of symptom onset. Our multidisciplinary stroke committee previously established the 12hour window accounting for 3 key considerations: prioritizing sensitivity over specificity, availability of resources enabling treatment beyond 4.5 hours of symptoms in select cases, and institutional research protocols.

We excluded patients who presented via EMS with prehospital stroke activation because no data existed with which to determine timeliness of stroke recognition before hospital arrival. All other patients in the stroke registry were activated by a member of the ED staff, after arriving either via EMS or by other means and were included in our investigation. Patient's presenting with symptoms concerning for stroke with symptom onset less than 12 hours were initially evaluated in the ED resuscitation rooms to facilitate rapid evaluation and care. Use of these rooms for potential stroke patients was determined by the evaluation of the triage nurse.

\subsection{Methods and measurements}

Trained research assistants, who were blinded to the study hypothesis, retrospectively reviewed the electronic health record (EHR) (ED PulseCheck, Optum Clinical Solutions, Inc., Eden Prairie, MN; Soarian, Cerner Corporation, North Kansas City, $\mathrm{MO}$; and OnBase, Hyland Software, Inc., Westlake, OH) for each patient in the registry to validate the registry data and abstract the following fields (determined a priori) using standardized abstraction forms: age, gender, triage emergency severity index (ESI) score [25], mode of arrival (EMS versus non-EMS), whether the patient was initially seen in an ED resuscitation/critical care room, initial vital signs (heart rate, respiratory rate, systolic blood pressure, diastolic blood pressure, and oxygen saturation), supplemental oxygen use and delivery method (none, nasal cannula, face mask, bag-mask ventilation, or intubated), Glascow coma scale (GCS) score, level of orientation (person, place, and time-range of 0-3), National Institute Health Stroke Scale (NIHSS) score, initial blood glucose value, elapsed time since the patient was last known to be at their baseline neurologic condition, previous history of stroke or transient ischemic attack, previous history of diabetes mellitus, and previous history of hypertension.

The EHR was also reviewed to determine whether the ED team documented treatment of another emergent, life-threatening condition that delayed care, categorized as airway/breathing intervention required, hypertension, hypotension, hypoglycemia, emergent electrolyte abnormality, or more than one of the above conditions. However, all documented delays pertained to the interval between stroke team activation and neuroimaging, and there were no documented delays in stroke recognition or activation. The registry and our EHR considered gender to be binary and self-reported by the patient. Rare missing values in the registry were obtained from the EHR by the abstractor. A second investigator independently searched the EHR for missing values after the initial abstraction and also independently validated all abstracted data for a subset of cases primarily abstracted by each research assistant. Missing values not available in either the registry or the EHR (vital signs, $\mathrm{n}=$ 1; glucose, $n=4$; and GCS, $n=75$ ) were replaced with the corresponding median value for the remaining data set. One entry in the registry was an exact duplicate, so the affected patient was analyzed only once. We have previously validated and employed a similar abstraction and data verification methodology for another registry-based study [6].

\subsection{Outcomes}

Our primary outcome was DTA time, defined as the interval between the EHR timestamp for ED arrival and the time of institutional stroke team activation by the ED staff, as recorded in the registry. We chose DTA given its prevalence in the literature as a key step in ED stoke care [5, 8, 11, 23, 24, 26] and its inherent threshold effect on other metrics, such as doorto-imaging time and door-to-needle time for thrombolytics. In our primary analysis, we considered DTA as a continuous outcome. In a secondary analysis, we considered the binary outcome of DTA $\leq 15$ minutes (the national goal). Patients for whom the documented duration of symptoms was shorter than the DTA time (one possible explanation being that symptoms may have begun while already in the ED) were reviewed for potential exclusion by full-text review of the EHR documentation by a senior investigator, but no cases of documented symptom onset while in the ED were identified in the included population.

Our secondary outcome was the binary variable of DIT within the national goal (at the time of the study) of $\leq 25$ minutes [4], defined as the interval between ED arrival and 
TA B L E 1. Discrete predictor variables and study subject characteristics.

\begin{tabular}{lcccc} 
Discrete Predictor & All Patients (n=211) & Women (n=117) & Men ( $=94)$ & $\begin{array}{c}P \text { Value for } \\
\text { Gender Difference }\end{array}$ \\
& $\mathrm{n}(\%)$ & $\mathrm{n}(\%)$ & $\mathrm{n}(\%)$ & \\
Gender & $117(55.5)$ & $117(100)$ & $0(0)$ & N/A \\
$\begin{array}{l}\text { Supplemental oxygen (intubated, high-flow, or } \\
\text { non-rebreather mask versus nasal cannula or none) }\end{array}$ & $11(5.2)$ & $5(4.3)$ & $6(6.4)$ & 0.54 \\
History of diabetes mellitus & $65(30.1)$ & $33(28.2)$ & $32(34.0)$ & 0.37 \\
History of hypertension & $138(65.4)$ & $74(63.2)$ & $64(68.1)$ & 0.47 \\
History of stroke/TIA & $69(32.7)$ & $35(29.9)$ & $34(36.2)$ & 0.38 \\
Orientation level (<3) & $57(27.0)$ & $32(27.4)$ & $25(36.6)$ & 1.00 \\
GCS score (<14) & $29(13.7)$ & $14(12.0)$ & $15(16.0)$ & 0.43 \\
ESI score (1 or 2) & $127(60.2)$ & $67(57.3)$ & $60(63.8)$ & 0.40 \\
Mode of arrival (EMS) & $115(54.5)$ & $67(57.3)$ & $51(54.3)$ & 0.41 \\
Resuscitation room used & $48(22.7)$ & $23(19.7)$ & $25(26.6)$ & 0.25 \\
\hline
\end{tabular}

initial neuroimaging completion (non-contrast computerized tomography at our institution) with images available for interpretation [1]. Additional secondary clinical endpoints were admission to a neurology service, final ED diagnosis of stroke or intracranial hemorrhage, and administration of intravenous thrombolytics or neurointerventional procedure, which were recorded directly in the registry and verified in the EHR.

\subsection{Analysis}

Our primary predictor of interest was patient gender. We identified 18 additional candidate covariates by investigator consensus, which are listed in Table 1 and Table 2, based upon their plausibility as confounders and/or inclusion in prior studies. We also considered arrival hour of day and day of week as temporal effects but did not find an effect of month/year. We calculated mean arterial pressure as $1 / 3$ times systolic blood pressure plus $2 / 3$ times diastolic blood pressure and used it in lieu of including both systolic and diastolic values to reduce dimensionality. Because NIHSS and age had nonlinear associations with DTA, we performed a priori recursive partitioning analyses to identify potential cut-points to further reduce dimensionality and collapse these nonlinear continuous effects to discrete predictors.

Our primary analysis of continuous DTA was a multivariable Cox Proportional Hazards regression to account for the highly skewed distribution typical of time-to-event data, which typically violate many assumptions of ordinary least squares regression. The Cox model was based upon a non-parametric hazard function, defined as the conditional probability of a given patient achieving DTA in the next instant (i.e. their stroke symptoms being recognized), provided their stroke had not been recognized by that point. ("Hazard" is a misnomer in this context, where it is desirable to have a shorter timeto-event, and the term used in its statistical definition, not it's colloquial meaning.) For each predictor, we calculated $\mathrm{HR}$, the ratio of hazard rates per unit change in a covariate. We verified the Cox proportionality assumption by generating time-dependent covariates using interactions between each predictor and log-transformed DTA. With the exception of high flow oxygen, none of the time dependent covariates were significant. For high flow oxygen, we graphically verified that Kaplan-Meier curves were parallel across both levels of the covariate and concluded that the very small number of patients with high flow oxygen (causing wide confidence intervals for this covariate) were not likely to meaningfully jeopardize our overall analysis.

Given the prevalence of logistic regression in the existing literature on this topic, we also fit a multivariable logistic regression model of DTA $\leq 15$ minutes with maximum likelihood estimation as a secondary analysis. We used an identical approach for our secondary analysis of DIT, except we also added ED occupancy rate [27] at the time of patient arrival, based on prior literature [6]. Given our desire to control for a number of plausible confounders, based mostly on prior research, we included all candidate variables in our model, regardless of univariate statistical significance. We assessed for baseline univariate differences between group using Fisher's Exact Test for categorical variables and Wilcoxon Rank Sums Test for continuous variables. The logistic regression model exhibited good overall fit (AUC 0.86 and Hosmer-Lemeshow lack-of-fit test $P=0.31$ ). Analyses were conducted using JMP Pro 13.2 (SAS Institute Inc., Cary, NC).

\section{Results}

\subsection{Characteristics of study subjects and baseline gender differences}

Among 490 consecutive stroke activation patients in the registry during the study period, 211 did not have pre-hospital stroke activation and were eligible for inclusion. Table 1 and Table 2 report the baseline characteristics of included patients, stratified by gender. We observed no statistically or clinically significant differences in baseline characteristics by gender, except that male patients tended to be slightly younger (Table 2).

Among all patients, DTA ranged from less than 1 minute to 
T A B L E 2. Continuous predictor variables and study subject characteristics.

\begin{tabular}{lcccc} 
Continuous Predictor & All Patients $(\mathrm{n}=211)$ & Women $(\mathrm{n}=117)$ & Men $(\mathrm{n}=94)$ & $\begin{array}{c}\text { P Value for } \\
\text { Gender Difference }\end{array}$ \\
& Median (IQR) [Range] & Median (IQR) & Median (IQR) \\
Age (years) & $66(23)[26-98]$ & $69(24)$ & $62(22)$ & 0.02 \\
Blood glucose (mg/dL) & $114(43)[62-971]$ & $112(40)$ & $117(45)$ & 0.04 \\
Blood pressure-systolic (mmHg) & $148(37)[73-232]$ & $148(29)$ & $149(42)$ & 0.74 \\
Blood pressure-diastolic (mmHg) & $84(22)[31-172]$ & $83(23)$ & $85(25)$ & 0.63 \\
Heart rate (min $\left.{ }^{-1}\right)$ & $80(20)[37-153]$ & $80(18)$ & $78(22)$ & 0.23 \\
NIHSS (1-42 points) & $2(5)[0-25]$ & $2(5)$ & $3(5)$ & 0.76 \\
Oxygen saturation (\%) & $97(3)[69-100]$ & $98(3)$ & $97(2)$ & 0.30 \\
Respiratory rate (min $\left.{ }^{-1}\right)$ & $18(4)[9-35]$ & $18(4)$ & $18(4)$ & 0.41 \\
$\begin{array}{l}\text { Time since patient last known to be at baseline } \\
\text { neurologic condition (hours) }\end{array}$ & $2.5(4.9)[0.5-12]$ & $2.6(5.0)$ & $2.0(4.4)$ & 0.60 \\
\hline
\end{tabular}

$I Q R$, interquartile range.

3 hours, 37 minutes (median 19 minutes, interquartile range [IQR] 23 minutes, $42.7 \% \leq 15$ minutes). For women, the range was less than 1 minute to 3 hours, 37 minutes (median 22, IQR 26 minutes). For males, less than 1 minute to 1 hour, 32 minutes (median 14, IQR 22 minutes). 53\% of males achieved DTA within the goal of 15 minutes, compared to $34 \%$ of women ( $P=0.008$ for two-tail univariate difference).

DIT ranged from 10 minutes to 3 hours, 41 minutes (median 36 , IQR 25 minutes) among all patients and was 10 minutes to 3 hours, 41 minutes (median 36, IQR 27 minutes) for women and 13 minutes to 1 hour, 56 minutes (median 33, IQR 25 minutes) for males. DIT was within the 25 -minute goal for $26 \%$ of patients overall, $35 \%$ for males and $18 \%$ for women $(P$ $=0.007$ for univariate difference).

\subsection{Main results}

Table 3 reports the HRs for each variable in the multivariable Cox model of DTA. The interpretation of HRs in this context is, "controlling for covariates, what is the probability of a patient with a specific characteristic achieving DTA time faster than a patient without that characteristic?" Being a woman was associated with an adjusted HR of 0.60 (95\% confidence interval 0.43 to 0.83 ) for timely recognition of stroke symptoms and activating stroke resources, compared to male gender. The HR for women in an unadjusted model was 0.73 (95\% confidence interval 0.55 to 0.96 ).

\subsection{Secondary outcomes}

Logistic regression models of DTA $\leq 15$ minutes and DIT $\leq$ 25 minutes had good overall fit (area under the curve 0.86 and 0.88 , respectively). Odds ratios for each variable are given in Table 4. Being a woman was a significant predictor of delays in both DTA and DIT.

There were no gender differences in the secondary clinical outcomes. The final ED diagnosis was stroke or intracranial hemorrhage in $66(31 \%)$ of patients; $128(61 \%)$ were admitted to a neurology service, and $7(3.3 \%)$ received thrombolytics or a neurointerventional procedure. Non-stroke diagnoses were varied and consistent with prior reports [21].

\section{Discussion}

Our investigation showed that door-to-stroke team activation and door-to-CT imaging were adversely affected by patient gender among patients without a pre-hospital stroke activation, when controlling for a broad list of potential confounders including ESI score and triage to critical care rooms. We observed gender differences in ESI, arrival method, and triage to critical care beds, all of which have been suggested as potential factors contributing to the gender disparity in stroke care. However, when controlling for these factors in our analysis, the gender difference in time to CT imaging remained.

This provides evidence to support suggestions in prior reports that triage factors may contribute to the gender disparity in time to CT imaging for acute stroke [5, 8]. Rose et al. did account for severity upon presentation using documented presumptive diagnosis at arrival, which could be seen as a proxy for triage severity, but actual ESI score and other triage factors were not considered. Kelly et al. did not account for specific triage processes in their analysis but acknowledged that the disparity in care appears to arise soon after arrival in the ED [5]. Our results confirm these prior suggestions and, more importantly, suggest that even when accounting for the confounding factors of ESI score, arrival method, and triage method, a gender disparity still exists. Although we did include an extensive list of potential confounders in our analysis, there are other potential confounders that were not included in our database and may have impacted the results such as race, socioeconomic status, anticoagulant use and history of atrial fibrillation.

Our results do contrast the findings of Madsen et al., who did not find a gender difference in CT timing, ESI score, or triage method [22]. They acknowledge that the academic setting of their facility combined with adherence to stroke protocols may have reduced disparities at their center, making the results less generalizable. Others have documented persistent gender differences in quality of care in non-academic settings [14]. 
TA B L E 3. Multivariable Cox Proportional Hazard Ratios for Door to Activation Time.

\begin{tabular}{ll} 
Gender (women vs. men) & $0.60(0.43-0.83)$ \\
Heart rate (per 10 min-1 increase) & $1.01(1.00-1.02)$ \\
NIHSS ( 2 vs. $<2$ ) & $1.47(1.05-2.05)$ \\
\hline ESI score (1-2 vs. 3-5) & $1.48(1.07-2.07)$ \\
Resuscitation room used & $2.86(1.76-4.64)$ \\
History of stroke/TIA & $0.80(0.57-1.11)$ \\
History of diabetes mellitus & $0.89(0.61-1.27)$ \\
\hline Orientation level (<3 vs. 3) & $0.91(0.57-1.43)$ \\
\hline History of hypertension & $0.95(0.65-1.40)$ \\
\hline Supplemental oxygen (intubated, high-flow, or non-rebreather mask vs. nasal cannula or none) & $0.96(0.38-2.30)$ \\
Time since patient last known to be at baseline neurologic condition (per 1 hour) & $0.97(0.93-1.01)$ \\
\hline Oxygen saturation (per 10\% increase) & $0.98(0.94-1.04)$ \\
Respiratory rate (per 1 min increase) & $0.99(0.94-1.04)$ \\
Blood glucose (per 10 mg/dL increase) & $1.00(1.00-1.00)$ \\
Age (per 1 year increase) & $1.00(0.99-1.01)$ \\
Mean Arterial Pressure (per 10 mmHg increase) & $1.00(0.99-1.01)$ \\
Mode of arrival (EMS vs. non-EMS) & $1.22(0.81-1.87)$ \\
GCS score (<14 vs. 14) & $1.72(0.88-3.23)$
\end{tabular}

$H R$ are the ratio of the hazard function between levels of a covariate, defined as the conditional probability of a given patient achieving DTA in the next instant (i.e. their stroke symptoms being recognized), provided their stroke had not been recognized by that point.

$H R=1$ implies no difference in DTA, and HR $<1$ implies proportionally slower DTA.

All figures are given as HR (95\% confidence interval).

Our study, however, was also conducted at an academic tertiary care facility geographically proximate to the site studied by Madsen et al. It is unclear why the two studies found contrasting results. We surmise this may be a result of varying internal processes between the two sites and differences in analytic approaches. Their study also included pre-hospital activations, making the two study populations unique. This highlights an important limitation of all similar studies to date; ours and others are single-center studies.

Our investigation has other notable limitations. We selected only patients without pre-hospital stroke activation because, by definition, DTA is zero in the registry if stroke resources were activated prior to ED arrival. We had no means to assess the speed with which EMS recognized stroke symptoms prior to arrival, and therefore, we had no way to evaluate for gender differences in this cohort. Excluding these patients creates a selection bias, in which included patients may have had more subtle, complex, or confusing presentations (despite controlling for NIHSS and other confounders) and may not be representative of the general population of potential stroke patients. This may explain why a relatively small proportion of included patients met DTA or DIT goals. As there was not a gender difference in the proportion of patients with prehospital activation, it is possible that our observed gender differences reflect a disparity only among the subgroup of potential stroke patients who went unrecognized by EMS or self-presented to the ED. However, mode of arrival was not a significant predictor in either our Cox or logistic models. It is possible that no disparity exists in those patient with a prehospital stroke activation.

Our primary analysis also assumed that the effect of gender on DTA is proportional over the range of observed DTA times. Our analysis did not account for the possibility of time-varying differential effects of gender. Our secondary logistic models were also sizable, and logistic regression performance may be diminished when there are a large number of predictors, relative to positive outcomes [28]. Stepwise regression techniques may obviate some of these problems but introduce others. We chose to include all available plausible predictors, which increases the risk of overfitting and potentially Type I error, but the similarity in findings between our primary and secondary analyses suggests that our observed gender difference in DTA is real.

Another possibility to be considered is that the severity of stroke presentation may, itself, exhibit a gender difference. While we did not find gender differences in initial NIHSS score, use of thrombolytics, or final diagnosis of stroke, our registry did not account for stroke territory, stroke subtype or initial presenting symptoms (other than NIHSS score), which could have contributed to delays in stroke recognition or activation $[29,30]$. Previous literature has hypothesized a gender differences in stroke presentation $[18,29]$ and stroke severity [31], but the disparity in timeliness of stroke care was not attributable to differences in clinical presentation [18], and we did not account for it in our study. Previous literature has also demonstrated gender differences in stroke sub-type 
TA B L E 4. Multivariable Logistic Regression Results for Door to Activation and Door to Imaging.

Odds Ratio for DTA $\leq 15$ minutes Odds Ratio for DIT $\leq \mathbf{2 5}$ minutes

\begin{tabular}{|c|c|c|}
\hline Gender (women vs. men) & $0.26(0.12-0.58)$ & $0.19(0.7-0.51)$ \\
\hline Heart rate (per $10 \mathrm{~min}^{-1}$ increase) & $1.36(1.05-1.79)$ & $1.3(0.97-1.82)$ \\
\hline NIHSS $(\geq 2$ vs. $<2)$ & $3.96(1.72-9.13)$ & $1.88(0.73-4.87)$ \\
\hline ESI score (1-2 vs. $3-5)$ & $3.10(1.29-7.42)$ & $4.01(1.38-11.68)$ \\
\hline Resuscitation room used & $8.33(2.59-26.78)$ & $14.31(3.50-58.48)$ \\
\hline History of stroke/TIA & $0.49(0.21-1.11)$ & $0.76(0.29-2.04)$ \\
\hline History of diabetes mellitus & $0.83(0.33-2.10)$ & $0.44(0.13-1.46)$ \\
\hline Orientation level (<3 vs. 3 ) & $1.42(0.48-4.17)$ & $2.16(0.60-7.78)$ \\
\hline History of hypertension & $0.85(0.35-2.10)$ & $1.84(0.58-5.84)$ \\
\hline $\begin{array}{l}\text { Supplemental oxygen (intubated, high-flow, or } \\
\text { non-rebreather mask vs. nasal cannula or none) }\end{array}$ & $2.55(0.30-21.92)$ & $0.03(0.00-17.54)$ \\
\hline $\begin{array}{l}\text { Time since patient last known to be at baseline } \\
\text { neurologic condition (per } 1 \text { hour) }\end{array}$ & $0.96(0.87-1.06)$ & $0.89(0.79-1.01)$ \\
\hline Oxygen saturation (per 10\% increase) & $0.75(0.52-1.06)$ & $4.95(0.40-61.81)$ \\
\hline Respiratory rate (per $1 \mathrm{~min}^{-1}$ increase) & $0.94(0.84-1.05)$ & $1.03(0.90-1.18)$ \\
\hline Blood glucose (per 10 mg/dL increase) & $1.04(0.99-1.12)$ & $0.97(0.86-1.09)$ \\
\hline Age (per 1 year increase) & $1.00(0.97-1.02)$ & $0.99(0.96-1.02)$ \\
\hline Mean Arterial Pressure (per $10 \mathrm{mmHg}$ increase) & $1.22(1.05-1.79)$ & $0.97(0.78-1.22)$ \\
\hline Mode of arrival (EMS vs. non-EMS) & $1.18(0.43-3.34)$ & $2.15(0.60-7.66)$ \\
\hline GCS score $(<14$ vs. $\geq 14)$ & $0.63(0.13-2.96)$ & $0.40(0.06-2.57)$ \\
\hline ED occupancy rate (per $10 \%$ increase) & $\mathrm{N} / \mathrm{A}$ & $0.83(0.74-0.92)$ \\
\hline
\end{tabular}

DIT, door-to-imaging time; DTA, door-to-activation time. All figures are given as odds ratio (95\% confidence interval). ED occupancy ratio was not included in model of DTA because its inclusion is not supported by prior literature (see text).

[31], which was not controlled for in our study, and may be a potential contributing factor to the disparity seen in our investigation. Although we controlled for NIHSS score in our analysis, the study population had a low median NIHSS score. Therefore, although we identified a gender disparity in our study population, this may not persist in patients with higher NIHSS scores.

This study suggests that women with suspected stroke experience delays in initial emergency department stroke care when compared to male patients in patients without pre-hospital stroke activation. Although our study demonstrated this disparity despite controlling for a number of potential confounding factors, it was not designed to further elucidate what additional factors may be contributing to the observed gender disparity. Nonetheless, our results indicate that further research is warranted to direct strategies to reduce and eliminate gender disparity in acute stroke care. This further research will be critical to inform targeted process improvement initiatives. Education of ED providers and staff may increase awareness of this disparity as a first step to bridging this gender gap in care.

\section{Conclusions}

Gender disparities exist in door-to-activation (recognition) time and door-to-imaging time among ED patients presenting with acute stroke symptoms. Women have longer times, even when controlling for triage and operational factors.

\section{AUTHOR CONTRIBUTIONS}

All authors contributed to the study conception and design. Material preparation, data collection and analysis performed by Sean S Michael. The first draft of the manuscript was written by Evangelia Murray and all authors commented on previous versions of the manuscript. All authors read and approved the final manuscript.

\section{ETHICS APPROVAL AND CONSENT TO PARTICIPATE}

This study was approved by the Institutional Review Board (IRB) of the University of Massachusetts Medical School.

\section{ACKNOWLEDGMENT}

I would like to express gratitude to all those who helped during the preparation of this manuscript and to thank all the peer 
reviewers for their opinions and suggestions.

\section{FUNDING}

This research did not receive any specific grant from funding agencies in the public, commercial, or not-for-profit sectors.

\section{CONFLICT OF INTEREST}

The authors declare that they have no conflicts of interest.

\section{AVAILABILITY OF DATA AND MATERIALS}

The datasets may be obtained from the corresponding author upon reasonable request.

\section{REFERENCES}

[1] Jauch EC, Saver JL, Adams HP, Bruno A, Connors JJ, Demaerschalk BM, et al. Guidelines for the early management of patients with acute ischemic stroke. Stroke. 2013; 44: 870-947.

[2] Marler JR, Tilley BC, Lu M, Brott TG, Lyden PC, Grotta JC, et al. Early stroke treatment associated with better outcome: the NINDS rt-PA stroke study. Neurology. 2000; 55: 1649-1655.

[3] Morgenstern LB, Kissela BM. Stroke Disparities. Stroke. 2015; 46: 35603563.

[4] Centers for Disease Control and Prevention. Prehospital and hospital delays after stroke onset-United States, 2005-2006. Morbidity and Mortality Weekly Report. 2007; 56: 474-478.

[5] Kelly AG, Hellkamp AS, Olson D, Smith EE, Schwamm LH. Predictors of rapid brain imaging in acute stroke. Stroke. 2012; 43: 1279-1284.

[6] Reznek MA, Murray E, Youngren MN, Durham NT, Michael SS. Door-to-imaging time for acute stroke patients is adversely affected by emergency department crowding. Stroke. 2017; 48: 49-54.

[7] Jungehulsing GJ, Rossnagel K, Nolte CH, Muller-Nordhorn J, Roll S, Klein M, et al. Emergency department delays in acute stroke-analysis of time between ED arrival and imaging. European Journal of Neurology. 2006; 13: 225-232.

[8] Rose KM, Rosamond WD, Huston SL, Murphy CV, Tegeler CH. Predictors of time from hospital arrival to initial brain-imaging among suspected stroke patients: the North Carolina Collaborative Stroke Registry. Stroke. 2008; 39: 3262-3267.

[9] Smith MA, Lisabeth LD, Brown DL, Morgenstern LB. Gender comparisons of diagnostic evaluation for ischemic stroke patients. Neurology. 2005; 65: 855-858.

[10] de Ridder I, Dirks M, Niessen L, Dippel D. Unequal access to treatment with intravenous alteplase for women with acute ischemic stroke. Stroke. 2013; 44: 2610-2612.

[11] Knauft W, Chhabra J, McCullough LD. Emergency department arrival times, treatment, and functional recovery in women with acute ischemic stroke. Journal of Women's Health. 2010; 19: 681-688.

[12] Reeves MJ, Bushnell CD, Howard G, Gargano JW, Duncan PW, Lynch $\mathrm{G}$, et al. Sex differences in stroke: epidemiology, clinical presentation, medical care, and outcomes. The Lancet Neurology. 2008; 7: 915-926.

[13] Reeves MJ, Wilkins T, Lisabeth LD, Schwamm LH. Thrombolysis treatment for acute stroke: issues of efficacy and utilization in women. Women's Health. 2011; 7: 383-390.

[14] McDermott M, Lisabeth LD, Baek J, Adelman EE, Garcia NM, Case E, et al. Sex disparity in stroke quality of care in a community-based study. Journal of Stroke and Cerebrovascular Diseases. 2017; 26: 1781-1786.
[15] Reeves M, Bhatt A, Jajou P, Brown M, Lisabeth L. Sex differences in the use of intravenous rt-PA thrombolysis treatment for acute ischemic stroke: a meta-analysis. Stroke. 2009; 40: 1743-1749.

[16] Arnao V, Caso V. Sex-related differences of acute stroke unit care: results from the Austrian stroke unit registry. Women'S Health. 2014; 10: 487489.

[17] Bushnell CD, Hurn P, Colton C, Miller VM, del Zoppo G, Elkind $\mathrm{MSV}$, et al. Advancing the study of stroke in women: summary and recommendations for future research from an NINDS-Sponsored Multidisciplinary Working Group. Stroke. 2006; 37: 2387-2399.

[18] Gargano JW, Wehner S, Reeves MJ. Do presenting symptoms explain sex differences in emergency Department Delays among Patients with Acute Stroke? Stroke. 2009; 40: 1114-1120.

[19] Jungehulsing GJ, Rossnagel K, Nolte CH, Muller-Nordhorn J, Roll S, Klein M, et al. Emergency department delays in acute stroke - analysis of time between ED arrival and imaging. European Journal of Neurology. 2006; 13: 225-232.

[20] Madsen TE, Seigel TA, Mackenzie RS, Marcolini EG, Wira CR, Healy $\mathrm{ME}$, et al. Gender differences in neurologic emergencies part I: a consensus summary and research agenda on cerebrovascular disease. Academic Emergency Medicine. 2014; 21: 1403-1413.

[21] Tafreshi GM, Raman R, Ernstrom K, Meyer BC, Hemmen TM. Gender differences in acute stroke treatment: the University of California San Diego experience. Stroke. 2010; 41: 1755-1757.

[22] Madsen TE, Choo EK, Seigel TA, Palms D, Silver B. Lack of gender disparities in emergency department triage of acute stroke patients. The Western Journal of Emergency Medicine. 2015; 16: 203-209.

[23] Fonarow GC, Smith EE, Saver JL, Reeves MJ, Hernandez AF, Peterson $\mathrm{ED}$, et al. Improving door-to-needle times in acute ischemic stroke: the design and rationale for the American Heart Association/American Stroke Association's Target: Stroke initiative. Stroke. 2011; 42: 2983-2989.

[24] Ruff IM, Ali SF, Goldstein JN, Lev M, Copen WA, McIntyre J, et al. Improving door-to-needle times. Stroke. 2014; 45: 504-508.

[25] Mistry B, Stewart De Ramirez S, Kelen G, Schmitz PSK, Balhara $\mathrm{KS}$, Levin S, et al. Accuracy and reliability of emergency department triage using the emergency severity index: an international multicenter assessment. Annals of Emergency Medicine. 2017; 71: 581-587.e3.

[26] Xian Y, Xu H, Lytle B, Blevins J, Peterson ED, Hernandez AF, et al. Use of strategies to improve door-to-needle times with tissue-type plasminogen activator in acute ischemic stroke in clinical practice: findings from target: stroke. Circulation. Cardiovascular Quality and Outcomes. 2017; 10: e003227.

[27] McCarthy ML, Aronsky D, Jones ID, Miner JR, Band RA, Baren JM, et al. The emergency department occupancy rate: a simple measure of emergency department crowding? Annals of Emergency Medicine. 2008; 51: $15-12$.

[28] Harrell FE, Lee KL, Mark DB. Multivariable prognostic models: issues in developing models, evaluating assumptions and adequacy, and measuring and reducing errors. Statistics in Medicine. 1996; 15: 361-387.

[29] Labiche LA, Chan W, Saldin KR, Morgenstern LB. Sex and acute stroke presentation. Annals of Emergency Medicine. 2002; 40: 453-460.

[30] Lisabeth LD, Brown DL, Hughes R, Majersik JJ, Morgenstern LB. Acute stroke symptoms. Stroke. 2009; 40: 2031-2036.

[31] Arboix A, Cartanyà A, Lowak M, García-Eroles L, Parra O, Oliveres $\mathrm{M}$, et al. Gender differences and woman-specific trends in acute stroke: Results from a hospital-based registry (1986-2009). Clinical Neurology and Neurosurgery. 2014; 127: 19-24.

How to cite this article: Kevin A Kotkowski, Evangelia Murray, Martin A Reznek, Sean S Michael. Gender differences in time from arrival to stroke team activation in patients presenting with acute stroke symptoms. Signa Vitae. 2021;17(3):130-136. doi: $10.22514 /$ sv.2021.051. 